\title{
Enroll in the REMS
}

National Cancer Institute

\section{Source}

National Cancer Institute. Enroll in the REMS. NCI Thesaurus. Code C128504.

The healthcare provider or patient must enroll in the REMS Program. Enrollment is the process by which REMS participants provide basic identifying and demographic information to the REMS Program (e.g. through a paper form or a website), allowing the application holder to track participants and communicate with them. 